\title{
ESTUDIO ANATÓMICO DE LAS INSERCIONES DE LOS MÚSCULOS EXTENSORES RADIALES LARGO Y CORTO DEL CARPO
}

\author{
ANATOMICAL STUDY OF INSERTIONS OF THE EXTENSOR CARPI RADIALIS LONGUS AND BREVIS
}

\author{
"Mauricio Ferreira Caetano; ${ }^{* *}$ Walter Manna Albertoni; ${ }^{* * *}$ Edie Benedito Caetano \& ${ }^{* * * * *}$ Rodrigo Montaño Pérez
}

Caetano, F. M.; Albertoni, M. W.; CAetano, B. E. \& PÉREZ, M. R. Estudio anatómico de las inserciones de los músculos extensores radiales largo y corto del carpo. Int. J. Morphol., 22(4):245-251, 2004.

RESUMEN: En el análisis de los tendones de los músculos extensores radiales largo y corto del carpo, basados en la disección anatómica de 60 miembros superiores de cadáveres adultos de raza blanca y sexo masculino, registramos que las variaciones anatómicas no son muy frecuentes. La presencia de tendones accesorios ocurrió en apenas 3 manos disecadas, todas del músculo extensor radial largo del carpo. La presencia de vínculos tendinosos uniendo los tendones, fue registrada en 17 piezas. La presencia de un tendón intermedio uniendo los dos tendones, fue verificada en 4 piezas. La inserción del tendón del músculo extensor radial largo del carpo ocurrió, invariablemente, en el lado radial de la base del segundo metacarpiano y del músculo extensor radial corto en el lado radial de la base del tercer metacarpiano.

Basados en nuestras disecciones podemos afirmar que la conformación anatómica normal de los músculos extensores radiales largo y corto del carpo, es la presencia de un cuerpo muscular y un tendón de cada uno de los músculos extensores radiales que son cubiertos por los músculos abdutor largo y extensor corto; pasan por el segundo compartimiento dorsal del puño y se insertan, el músculo extensor radial largo en el lado radial de la base del segundo metacarpiano y el músculo extensor radial corto en el lado radial de la base del tercer metacarpiano, invariablemente.

PALABRAS CLAVE: 1. Anatomía; 2. Músculos extensor radial largo y corto del carpo; 3. Variación anatómica.

\section{INTRODUCCIÓN}

Analizando la literatura encontramos pocas publicaciones respecto a las variaciones anatómicas de los tendones de los músculos extensor radial largo y extensor radial corto del carpo.

El conocimiento y frecuencia de las variaciones anatómicas de los músculos extensores radiales largo y corto del carpo y de sus tendones tienen gran importancia clínica. Por ejemplo, en las secuelas de las lesiones del plexo braquial o de lesiones altas de los nervios del miembro superior, donde son necesarias múltiples transferencias tendinosas para dar alguna función a la mano, es posible utilizar el tendón del músculo extensor radial corto o largo del carpo duplicado para realizar dos funciones diferentes.

Otra situación de importancia clínica relevante es que, en todos los casos que disecamos, los tendones de los mús- culos extensores radiales largo y corto del carpo estaban unidos entre sí por alguna forma de conexión. Cuando esas conexiones ocurren a través de tejido areolar blando, como en la mayoría de los casos conforme está registrado en la Fig. 5, es posible realizar la transposición de los tendones por dos pequeñas incisiones. Una sobre la inserción en la base de los metacarpianos y otra a nivel de la unión miotendinosa, así se desinserta el tendón en la base del metacarpiano por la incisión distal y se tracciona el tendón por la incisión proximal, liberándolo de las conexiones blandas, para hacer su transposición y ejecutar la función deseada. En caso de unión por vínculo tendinoso único, como en la Fig. 7, o uniones múltiples, como en la Fig. 6, no es posible ese procedimiento por dos pequeñas incisiones, siendo necesaria una incisión amplia para deshacer los vínculos tendinosos y separar los tendones, para después efectuar la transferencia tendinosa. 


\section{MATERIAL Y MÉTODO}

El material de estudio de este trabajo estaba constituido por 60 piezas anatómicas, correspondientes a 30 pares de miembros superiores de cadáveres. De las 60 piezas, 20 correspondían a 10 cadáveres frescos conservados en refrigerador y 40 piezas a 20 cadáveres, que fueron preparados previamente, por inyección de formol al $10 \%$ y glicerina, en la arteria femoral.

El trabajo fue realizado en el Laboratorio de Anatomía de la Facultad de Medicina de la Pontífica Universidad Católica de São Pablo - PUC-SP. Los miembros disecados pertenecían a individuos con edades entre 23 y 67 años. Los cadáveres eran de raza blanca y sexo masculino.

Los cadáveres fueron colocados en la mesa de disección, en la posición de decúbito ventral, con el miembro superior en posición de abducción sobre una tabla de madera.

La disección siguió el siguiente orden: Se iniciaba por una incisión transversal a nivel de la cabeza de los metacarpianos. Otra incisión transversal fue realizada a nivel del codo.

El punto medio de cada una de esas incisiones transversales fue unido por una incisión longitudinal dorsal. De esta forma dos colgajos fueron levantados y rebatidos. Los músculos extensores radiales largo y corto del carpo fueron disecados desde su origen hasta la inserción de sus tendones. La presencia de variaciones anatómicas como la presencia de tendones accesorios, ausencia de tendones, duplicación de los tendones, alteraciones en el trayecto fueron observadas y anotadas. La inserción distal del tendón fue verificada minuciosamente y muchas veces con auxilio de lupa con aumento de dos veces.

\section{RESULTADOS}

Haciendo el análisis de los músculos extensores radiales largo y corto del carpo, desde su origen hasta su inserción, podemos afirmar que registramos la presencia de variaciones anatómicas significativas.

En tres de las 60 disecciones, siendo una bilateral, encontramos dos tendones que se originaban de un mismo músculo. En esas tres situaciones, la duplicación tendinosa fue del músculo extensor radial largo del carpo (Fig. 1). No verificamos esa alteración en el músculo extensor radial corto del carpo. Sin embargo, encontramos 20 veces la presencia de hendiduras separando parcialmente los tendones (Fig. 2). Estas hendiduras fueron verificadas en 14 ocasiones en el músculo extensor radial largo del carpo, y seis en el músculo extensor radial corto del carpo. En el trayecto de los tendones extensores radiales del carpo hasta su inserción, evidenciamos que los músculos abdutor largo y extensor corto del pulgar cruzaban oblicuamente sobre ellas. Actuando como poleas, fijando esos tendones a las estructuras óseas del antebrazo. En cuatro de 30 cadáveres disecados, encontramos bilateralmente la presencia del músculo abdutor largo del pulgar, ensanchado como si estuviera incorporado un músculo adicional, expandiéndolo lateralmente y proporcionándole un aumento de la amplitud, lo que creaba un sistema de túnel retinacular ampliado, donde se encajaban los dos músculos extensores radiales del carpo (Fig. 3). Esa disposición es interesante, pues actúa como un sistema retinacular accesorio, proximal al retináculo de los extensores, auxiliando los extensores radiales para mantenerse junto al plano óseo y con eso hacer más eficiente su acción extensora en el puño.

Los tendones de los músculos extensores radiales del carpo se apoyan sobre la extremidad distal del radio ocupando el segundo compartimiento formado por el retináculo de los músculos extensores. Removiendo esos tendones de su compartimiento, pudimos evidenciar la presencia de una pequeña cresta longitudinal separando dos canales. En el canal del lado radial pasa el tendón del músculo extensor radial largo del carpo y del lado ulnar pasa el tendón del músculo extensor radial corto del carpo (Fig. 4). En los casos que registramos la duplicación del tendón no estaban presentes crestas longitudinales separando los tendones duplicados en compartimientos separados.

Con relación a la inserción de los tendones, no hubo variaciones anatómicas. En todas las piezas encontramos la inserción del músculo extensor radial largo del carpo en el lado radial de la base del segundo metacarpiano y la inserción del músculo extensor radial corto del carpo en el lado radial de la base del tercer metacarpiano. Como la inserción ocurre en el lado radial de la base de los metacarpianos, la primera impresión puede ser de inserción en más de un metacarpiano; sin embargo, al profundizar la disección hasta el plano óseo, pudimos verificar que no existieron inserciones de los tendones en más de un metacarpiano, en ningún caso disecado.

Relativo al grosor de los tendones de los músculos extensores radiales largo y corto del carpo, no fue encontrada ninguna discrepancia significativa entre ellos. En todo su trayecto los tendones de los músculos extensores radiales se presentaban unidos por un tejido areolar, blando que podía deshacerse con facilidad con una simple maniobra traccionando un tendón para el lado radial y el otro para el lado ulnar (Fig. 5). Este hecho fue verificado en 45 piezas anatómicas. 

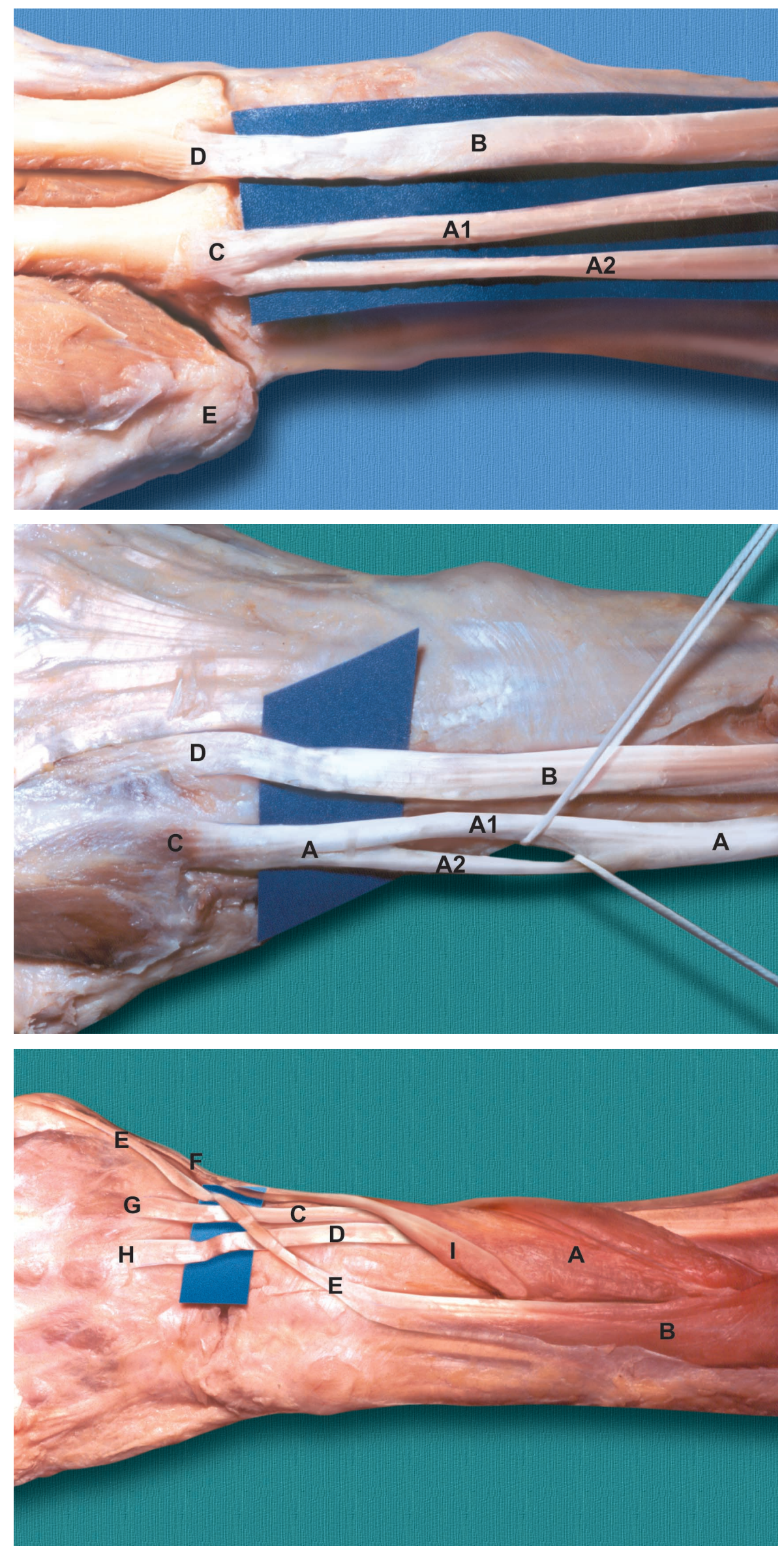

Fig. 1. Visión aproximada de la duplicación del tendón del músculo ERLC (músculo extensor radial largo del carpo) en A1 e A2) con la inserción de ambas en el lado radial de la BSM (base de segundo metacarpiano) (C). Tendón del músculo ERCC (músculo extensor radial corto del carpo) (B) insertado en el lado radial da BTM (base del tercer metacarpiano). BPM (base del primer metacarpiano) (E).

Fig. 2. Se observa un surco en el tendón del ERLC (A) provocando duplicación parcial del tendón. Tendón de músculo ERCC (B). BSM (C). BTM(D).

Fig. 3.Variación anatómica en la conformación del cuerpo muscular del ALP (músculo abductor largo del pulgar) (A) que se presenta expandido actuando como polea para el paso de los tendones ERCC (D), ERLC (C). Músculo ELP (músculo extensor largo del pulgar) (B) con su tendón (E). Músculo ECP (músculo extensor corto del pulgar) (I) con su tendón (F). BSM (G). BTM (H). 

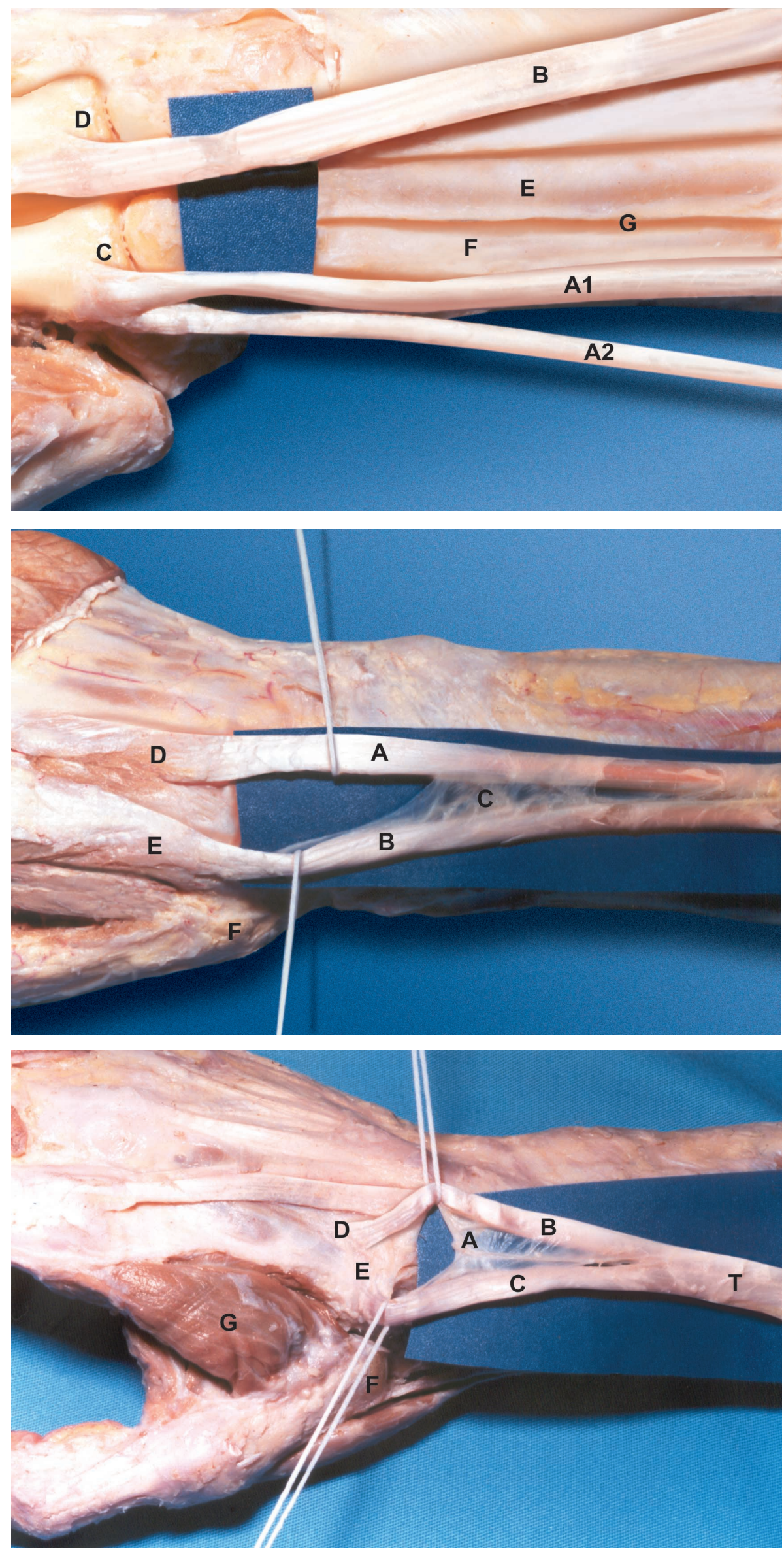

Fig. 4. En los casos de duplicación del tendón ERLC (A1 y A2) éstos, pasaban por un canal único $(\mathrm{F})$, separado por un septo $(\mathrm{G})$ del canal del ERCC (E). Tendón ERCC (B). BSM (C). BTM (D).

Fig. 5. Los tendones de los músculos ERCC (A) y ERLC (B) pueden estar unidos por un tejido areolar flojo (C). BTM (D). BSM (E). BPM (F).

Fig. 6. La presencia de vínculos tendinosos (A), que a diferencia del tejido areolar blando dificulta la separación de los tendones ERLC (C) y ERCC (B). BTM (D). BSM (E). BPM (F). Primer músculo interóseo dorsal $(\mathrm{G})$. 

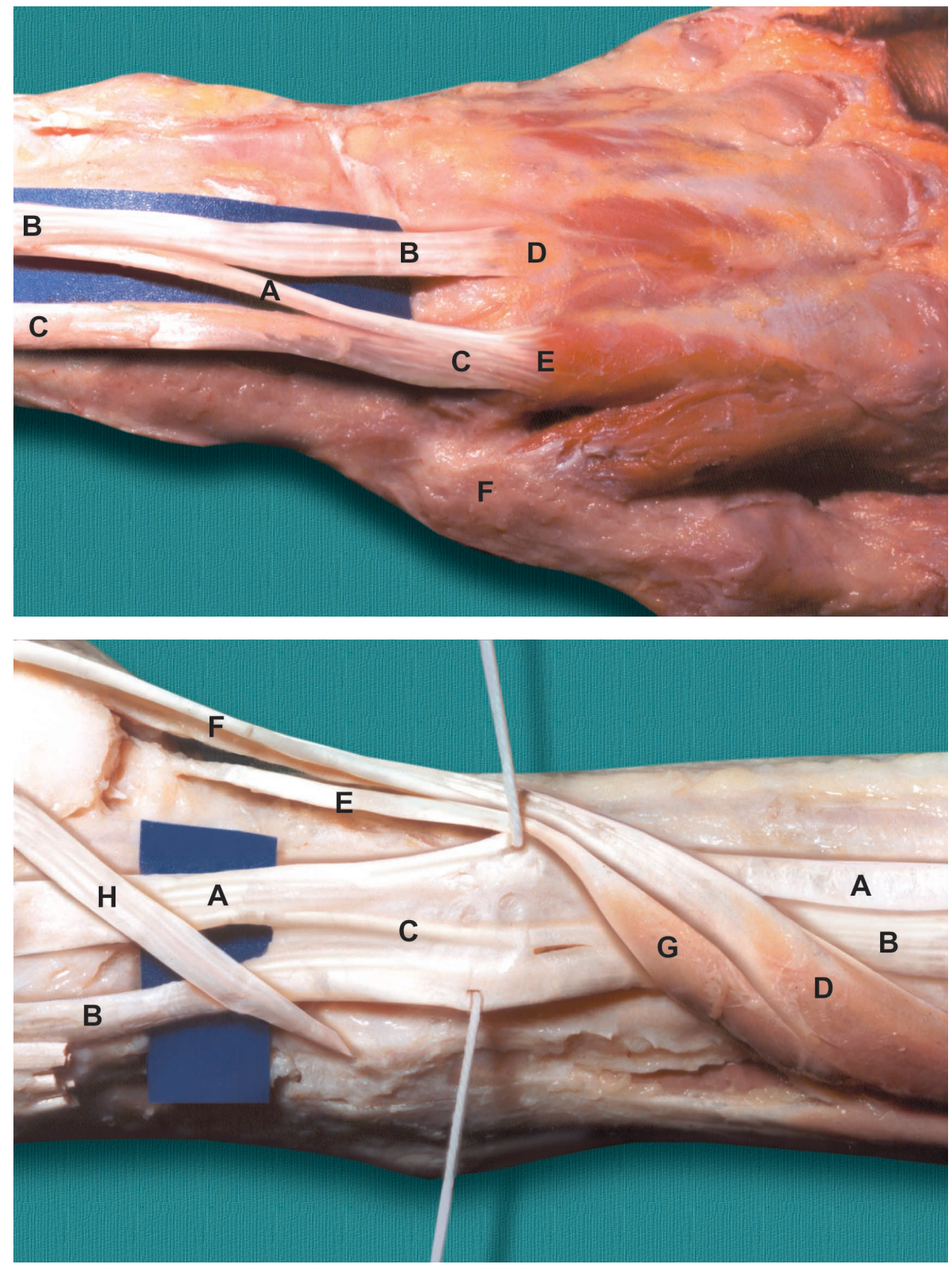

Fig. 7. Vínculo grueso y único pareciendo un tendón intermedio (A) uniendo los tendones de los músculos ERCC (B) y ERLC (C). BTM (D). $\operatorname{BSM}(\mathrm{D})$. BPM (F)
Fig. 8. Visión aproximada de los vínculos gruesos y resistentes (C) que dificultan la separación de los tendones del ERCC (A) y ERLC (B). Esa con formación no muy frecuente, dificulta el uso de los tendones en las transferencias tendíneas. Músculo ALP $(\mathrm{G})$ con su tendón (E). Músculo ECP (D) con su tendón (F). Tendón ELP (H).
La presencia de vínculos tendinosos uniendo esos tendones, fue encontrada en 17 de las 60 piezas anatómicas disecadas. La disposición de los vínculos tendinosos mostró gran variabilidad (Figs. 6, 7 y 8). En cuatro observaciones encontramos la presencia de un vínculo único y grueso uniendo los tendones como si fuera un tendón intermedio. En todas las situaciones ese tendón intermedio se dirigía del tendón del músculo extensor radial largo hacia el tendón del músculo del extensor radial corto y fue observado siempre próximo a la inserción distal de los tendones (Fig. 7).

\section{DISCUSIÓN}

Las variaciones anatómicas encontradas durante la disección de los músculos extensores radiales largo y corto del carpo, no fueron numerosas, pero si importantes desde el punto de vista clínico-quirúrgico.

Iniciando el análisis de esos músculos a partir de su origen, encontramos, invariablemente, la presencia de dos cuerpos musculares originando los tendones de los músculos extensores radiales largo y corto. La descripción de Khaledpour \& Schindelmeiser (1994) sobre la presencia bilateral de un músculo extensor radial del carpo accesorio, que se localizaba entre los músculos extensores radiales largo y corto del carpo 
y, se continuaba con dos tendones, que tenían inserción en la base del primero y segundo metacarpianos, no fue encontrada en nuestras disecciones. Tampoco fue verificada en nuestras piezas anatómicas, la presencia de un músculo encontrado por Testut (1884), que tenía origen común con los músculos extensores radiales largo y corto del carpo, continuaba con un tendón cubierto por el músculo braquiorradial, con trayecto similar al de los tendones de los músculos extensores radiales, yendo a insertarse en la base del primer metacarpiano, denominado este músculo, como abdutor humeral del pulgar. Kaplan (1961) afirmó que puede estar presente sólo un músculo extensor radial del carpo, que continúa con dos tendones o sólo con un tendón que se desdobla en lugar variable de su trayecto para insertarse en el segundo y tercer metacarpianos. No registramos esa variación anatómica en nuestras disecciones.

Los autores Fabrizio \& Clement (1996) relataron la presencia de un músculo abdutor largo, expandido lateralmente, formando un túnel retinacular, donde se encajan los tendones de los músculos extensores radiales largo y corto del carpo. Encontramos la presencia de esa variación en 8 antebrazos y concordamos que esa variación actúa como un sistema retinacular adicional, manteniendo los tendones junto al plano óseo y dejando más eficiente su acción como extensores del puño. La presencia de esa conformación anatómica dificulta abordajes de las fracturas del tercio distal del radio, pues puede ser necesaria la resección de parte del vientre muscular del abductor expandido, para facilitar la colocación de placas y tornillos.

En su análisis, Warwick \& Williams (1979) relataron que los tendones de los músculos extensores radiales largo y corto del carpo se pueden desdoblar y unirse nuevamente para insertarse, invariablemente, en la base del segundo y tercer metacarpianos. Esa observación fue verificada en veinte de nuestras disecciones, en la que los tendones se originaban del músculo siempre como un tendón, se desdoblaban por algunos centímetros, uniéndose nuevamente, conforme a la Fig. 2. No podemos considerar esa conformación como tendones accesorios, ya que se trata tan sólo de una duplicación parcial de un tendón, que se presentaba único tanto en su origen como en su inserción.

Autores como Hollinshead (1958), Kaplan, Goss (1977) relataron que los tendones de los músculos extensores radiales largo y corto del carpo se pueden dividir bien cerca de su inserción e insertarse en la base de más de un metacarpiano. Otros autores como Le Double (1897) y Testut \& Latarjet (1947) señalaron que el músculo extensor radial largo del carpo puede insertarse en la base del primero y segundo metacarpianos, y el tendón del músculo extensor radial corto del carpo en la base del segundo y tercer metacarpianos. En nuestras preparaciones no encontramos la inserción de ninguno de los músculos extensores radiales del carpo en más de un metacarpiano. La explicación que tenemos para ese hecho, es que la inserción del tendón del músculo extensor radial largo del carpo ocurre en un tubérculo en el lado radial de la base del segundo metacarpiano y el tendón del músculo extensor radial corto no se realiza en el centro de la base del tercer metacarpiano, pero sí, de forma bastante acentuada, en lado radial de su base. Como las bases de los metacarpianos están fuertemente unidas entre sí, puede aparentar que la inserción ocurre en la base de dos metacarpianos; sin embargo si profundizamos la disección hasta el plano óseo, queda claro que la inserción está en el lado radial de la base de uno de los metacarpianos. Ese hecho es evidente en la Fig. 4.

La discrepancia entre el diámetro de los dos tendones de los músculos extensores radiales del carpo no fue mencionada por los autores investigados, tampoco evidenciamos ese hecho; en algunos cadáveres sólo observamos un leve predominio del grosor del tendón del músculo extensor radial corto del carpo con relación al tendón del músculo extensor radial largo.

La presencia de vínculos tendinosos entre los tendones de los músculos extensores radiales largo y corto del carpo no es rara. Hollinshead afirma que la frecuencia vínculos tendinosos entre los tendones extensores radiales, es significativa, pero no refiere en qué porcentaje ocurren. En 43 de las manos disecadas, no encontramos la presencia de vínculos tendinosos entre los tendones de los músculos extensores radiales del carpo, de tal forma que traccionando los tendones con una fuerza pequeña, era posible separarlos fácilmente, inclusive para remover el tejido retinacular areolar blando que los juntaba, como puede ser observado en la Fig. 5.

En 17 piezas encontramos la presencia de vínculos tendinosos, alguno muy delgados, otros más gruesos uniendo los dos tendones de manera que era necesaria la aplicación de fuerza desproporcional para romper esas conexiones tendinosas, que pueden ser vistas en la Fig. 8. Esas particularidades que nos permiten separar los tendones con bastante facilidad, que encontramos en 43 de nuestras disecciones, tienen interesante importancia clínica, pues nos permiten, con pequeñas incisiones proximal y distal, separar los tendones cuando pretendemos utilizar uno de éstos para realizar una transposición tendínea. La presencia de vínculas entre ellos, exige la exposición total de los tendones para seccionar esas conexiones. 
En 4 de nuestras disecciones, encontramos la presencia de un vínculo único y grueso uniendo los tendones. Testut \& Latarjetdescribieron esos vínculos con el nombre de tendón intermedio. En cuatro observaciones, ese tendón se dirigía desde el tendón del músculo extensor radial largo para el tendón del músculo extensor radial corto del carpo y fue observado siempre cerca a la extremidad distal del tendón, como puede ser mostrado en la Fig. 7. Testut \& Latarjet afirmaron que algunas veces ese tendón intermedio puede aislarse completamente de los tendones de los músculos extensores radiales del carpo. No fue posible ese aislamiento en nuestras disecciones.
Autores como Tandler (1928), Rouvière (1948), Hollinshead, Kaplan y Gardner \& Osburn (1980) relataron la presencia de un tendón extensor radial accesorio, pero no mencionaron con qué frecuencia aparece. Testut \& Latarjet citan que Wood encontró en, 6 de 175 disecciones, ese tendón accesorio. Nosotros verificamos en 3 de nuestras 60 disecciones, tendones originándose del músculo extensor radial largo del carpo, pero en ningún caso el origen de los dos tendones se realizó del músculo extensor radial corto del carpo. Ese tendón accesorio se insertaba al lado del tendón del músculo extensor radial largo del carpo en el lado radial de la base del segundo metacarpiano, como se observa en la Fig.1.

CAETANO, F. M.; ALBERTONi, M. W.; CAETANO, B. E. \& PÉREZ, M. R. Anatomical study of insertions of the extensor carpi radialis longus and brevis. Int. J. Morphol., 22(4):245-251, 2004.

SUMMARY:The aim of this paper was to analyse the anatomical variations of the extensor carpi radialis longusand brevis in sixty male adults cadaver upper limbs.

The extensor carpi radialis longus and brevis muscles and tendons had a few anatomical variations, but very important about surgical aspects. The supernumerary tendons were found in 3 hands (all in relation to the extensor carpi radialis longus tendon). The presence of tendinous connections between the two tendons was found in 17 upper limbs. The presence of an accessory tendon making the union between the tendons of extensor carpi radialis brevis and longus was registered in 4 dissected hands. The insertion of the extensor carpi radialis longus tendon was in the radial side of the base of second metacarpal bone and the extensor carpi radialis brevis in the radial side of the base of third metacarpal bone in all dissected hands.

The more common pattern of the extensor carpi radialis longus and brevis was one muscle and one tendon of the extensor carpi radialis longus and brevis, localized in the second dorsal wrist compartment, and with a constant insertion in the radial side of bases of the second and third metacarpal bones.

KEY WORDS: 1. Anatomy; 2.Extensor carpi radialis longus and brevis muscles; 2. Anatomical variations.

\section{REFERENCIAS BIBLIOGRÁFICAS}

Fabrizio, P. A. \& Clement, F. R. A variation in organization of abdutor pollicis longus. Clin. Anatomy, 9(6): 371-5; 1996

Gardner, W. D. \& Osburn, W. A. Anatomia do corpo humano. 2. ed. São Paulo, Atheneu, 1980. pp 200-5

Goss, C. M. Gray Anatomia. 29a ed. Rio de Janeiro, Guanabara Koogan, 1977. pp 379-88,

Hollinshead, W. H. Anatomy for surgeons. New York, Hoeber Harper, 1958. pp393-94.

Kaplan, E. B. Anatomia funcional y quirurgica de la mano. Buenos Aires, Artecnica, 1961. pp 101-43,

Khaledpour, C. \& Schindelmeiser, J. Atypical course of the rare acessory extensor carpi radialis muscle. J. Anatomy, 184: $161-3 ; 1994$.

Le Double, A. F. Traité des variations do système musculaire de l'homme. Paris, Schleicher, 2:103-71, 1897.

Rouvière, H. Anatomie Humaine. 6. ed. Masson, Paris, 1948. V. 2. pp 227-43,
Tandler, J. Tratado de anatomia sistemática. 2. ed. Barcelona, Salvat, 403-4, 1928. V. 1.

Testut, L. \& Latarjet, A. Tratado de Anatomía Humana. 8a. ed. Barcelona, Salvat, 1947. V. 1. pp. 1059-86.

Testut, L. Les anomalies musculaires chez l'homme expliquée par l'anatomie comparée. Paris, Masson, 1884. pp 947-77

Warwick, R. \& Williams, P. L. Gray Anatomia. 3a. ed. Rio de Janeiro, Guanabara-Koogan, 1979. V. 1. pp 520-7.

Direccion para correspondencia:

Prof. Dr. Walter Manna Albertoni

Departamento de Ortopedia y Traumatologia

Universidade Federal de São Paulo - Escola Paulista de Medicina

Rua Botucatú 740, Vila Clementino

CEP 04023-900

São Paulo - SP

BRASIL
Recibido: 06-07-2004

Aceptado: 10-09-2004 